\title{
Leder, Dietrich/Nieland, Jörg-Uwe/Schaaf, Daniela: Die Entstehung des Mediensports. Zur Geschichte des Sportdokumentarfilms.
}

\author{
Köln: Herbert von Halem 2020. 360 Seiten. Preis: $€$ 35,99 \\ (e-book)
}

\author{
Alexis von Mirbach
}

Angenommen: 6. März 2021 / Online publiziert: 24. März 2021

(C) Der/die Autor(en) 2021

Seit den ersten filmischen Aufnahmen Ende des 19. Jahrhunderts zählen Bilder von Sportereignissen zu den Treibern der Medienevolution. Derzeit erleben Sportdokumentarfilme bei Streaming-Dienstleistern und Videoplattformen einen Boom und gewannen im vergangenen Jahrzehnt mehrfach den Dokumentarfilmpreis bei den Academy Awards (Oscars). Dennoch ist die Thematik in der wissenschaftlichen Literatur bislang nur randständig behandelt worden. Dietrich Leder, Jörg-Uwe Nieland und Daniela Schaaf schließen diese Forschungslücke, indem sie die erste Monografie im deutschsprachigen Raum (und je nach Definition auch international) verfasst haben, die ausschließlich Sportdokumentarfilme behandelt. Ein Ziel der Autoren ist, eine Systematisierung des Genres aus historischer Perspektive vorzulegen, was eindrucksvoll gelingt. Die Geschichte des Sportdokumentarfilms ist zugleich eine Geschichte der Sport- und Gesellschaftsentwicklung. Die Wechselwirkungen mit Leistungssport, Politik, Wirtschaft, Publizistik, Film, Technik sowie relevanten gesellschaftlichen Themen wie Sexismus, Integration oder Doping fassen die Autoren unter der Klammer der Medialisierung zusammen. Das „Alleinstellungsmerkmal“ (S. 16) des Bandes sei die Analyse der Sportdokumentation im Zusammenhang mit der Medialisierung; ihrem „Anspruch“ (ebd.) werden Leder, Nieland und Schaaf weitgehend gerecht.

Das theoretische Fundament ist im zweiten Teil interdisziplinär und kenntnisreich gelegt. Nieland stellt aus einem sportwissenschaftlichen Blickwinkel die Geschichte des modernen Sports dar, die er um die Geschichte der Sportpublizistik ergänzt. Leder behandelt aus filmwissenschaftlicher Perspektive die Historie des Dokumentarfilms - erst allgemein, anschließend explizit auf den Sport bezogen. Schaaf zeigt

\footnotetext{
Dr. A. von Mirbach $(\bowtie)$

Institut für Kommunikationswissenschaft und Medienforschung, Ludwig-Maximilians-Universität München, Oettingenstr. 67, 80538 München, Deutschland

E-Mail: Alexis.Mirbach@ifkw.lmu.de
} 
nach einer Einführung in die Körpersoziologe auf Basis von Pierre Bourdieu und Michel Foucault, dass eine Analyse von Sportdokumentarfilmen ohne die Berücksichtigung des Körpers kaum möglich ist. So luzide die These hergeleitet ist, weist sie zugleich auf die Schwäche des Bandes hin: Es fehlt zum Abschluss des zweiten Teils eine Verknüpfung der Perspektiven zu einem stringenten Kategoriensystem für die anschließende Analyse der Filme, dem Herzstück der Publikation. So wird das Konzept der Medialisierung nicht definiert oder operationalisiert und auch die Körpersoziologie nicht in jedem Kapitel explizit behandelt (was sich der Leser nach der spannenden Einführung gewünscht hätte).

Die Auswahl der Filme basiert auf Bänden der Dokumentarfilmgeschichte, der Programme der Dokumentarfilm- und Sportfilmfestivals sowie Untersuchungen der Fernsehgeschichte. Um die Anzahl übersichtlich zu halten, werden nur ,Autorendokumentarfilme“ herangezogen, also Filme, bei denen ein Regisseur als verantwortliche Person ,dingfest gemacht“ (S. 13) werden kann. Die finale Auswahl erfolgt nach den Kriterien Relevanz, Vielfalt und Repräsentativität und mündet in zehn Kapiteln zu den Themenbereichen Olympische Spiele, Fußball-Weltmeisterschaften, NichtNarrative-Filme, Frauenfußball, Helden, Radsport (Doping), Skateboarding, Behindertensport, Akteure im Hintergrund sowie Zuschauer. In den einzelnen Kapiteln greifen sich die Autoren je ein bis drei Filme für eine ausführliche Analyse heraus und ergänzen diese um weitere Filme aus dem Feld. Dieses Vorgehen führt zur großen Stärke des Bandes: Die Autoren liefern Kontext! Produktionsgeschichten, Biografien zu Sportlern oder Regisseuren, Exkurse zu Sportarten, gesellschaftlichen, sportlichen oder medialen Entwicklungen und durchgehend weisen sie auf blinde Flecken hin - Dinge, die der Film ausgelassen hat, was im Fall des Radsportfilms Höllentour (Pepe Danquart 2004) aufgrund der Doping-Problematik ein ganzes Kapitel überschreibt. Durch die Kontextkategorien deckt jedes Kapitel seinen Themenbereich umfassend ab und inspiriert zu Thesen, die durch eine stärkere Verknüpfung der Kapitel untereinander mehr Raum zur Verallgemeinerung zuließe die dennoch nicht zu kurz kommt.

In Bezug auf die Medialisierung steht durch die Analyse der Dokumentarfilme als übergeordneter Befund, dass sich der moderne Sport und die Massenmedien schon immer wechselseitig beeinflusst haben. Partielle Schlussfolgerungen zur Medialisierung: Im Film Olympia. Fest der Völker. Fest der Schönheit (1938) wird deutlich, dass sich die Regisseurin Leni Riefenstahl früh Techniken bediente, die heute einer kommerziellen Medienlogik zugeschrieben werden: Sie begriff Sportwettbewerbe als „Halbprodukte“, die durch filmische Überhöhung mittels Dramatisierungen, Personalisierungen und Heroisierungen zu einem attraktiven Medienprodukt veredelt werden. When We Were Kings (Leon Gast 1996), ein Film über Muhammad Ali, dient als Ausgangspunkt, um verschiedene Formen von Helden-Geschichten zu reflektieren, die seit der Dualisierung des Rundfunks Mitte der 1980er-Jahre eine immer wichtigere Rolle spielen. Was bei Deutschland - ein Sommermärchen (Sönke Wortmann 2006) noch der Schlüssel für den Erfolg war, kommt 2014 in einem Dokumentarfilm über die deutsche Nationalmannschaft nicht mehr vor: die Nähe zu den Spielern. Der Grund: Durch die Social-Media-Kanäle benötigen die Stars die Kameras der Regisseure nicht mehr zur Vermarktung. Im Anschluss an Mein Weg nach Olympia - ein ziemlich behinderter Film (Niko von Glasow 2013) wird 
sichtbar, dass der Behindertensport durch Debatten über Schattenseiten wie „TechDoping“ (mit Prothesen) oder die Mordanklage gegen Oscar Pistorius die dringend benötigte mediale Aufmerksamkeit erhält. Die Verflechtung des Mediensports mit dem eSport führt zu einer Anpassung in der Realwelt, wenn sich etwa Lionel Messi auf dem Spielfeld so verhält, wie es ihm der „Computer-Messi“ vormacht.

Die Liste der Befunde zur Medialisierung ließe sich fortschreiben und verdeutlicht, dass Die Entstehung des Mediensports reich an feinen gesellschaftswissenschaftlichen Beobachtungen ist. Verknüpft mit den filmischen Anekdoten stellt der Band ein in der Wissenschaft nicht selbstverständliches Lesevergnügen dar. Wer in Deutschland zu Sportdokumentarfilmen forschen möchte, hat mit Leder, Nieland und Schaaf seinen Ausgangspunkt gefunden.

Funding Open Access funding enabled and organized by Projekt DEAL.

Open Access Dieser Artikel wird unter der Creative Commons Namensnennung 4.0 International Lizenz veröffentlicht, welche die Nutzung, Vervielfältigung, Bearbeitung, Verbreitung und Wiedergabe in jeglichem Medium und Format erlaubt, sofern Sie den/die ursprünglichen Autor(en) und die Quelle ordnungsgemäß nennen, einen Link zur Creative Commons Lizenz beifügen und angeben, ob Änderungen vorgenommen wurden.

Die in diesem Artikel enthaltenen Bilder und sonstiges Drittmaterial unterliegen ebenfalls der genannten Creative Commons Lizenz, sofern sich aus der Abbildungslegende nichts anderes ergibt. Sofern das betreffende Material nicht unter der genannten Creative Commons Lizenz steht und die betreffende Handlung nicht nach gesetzlichen Vorschriften erlaubt ist, ist für die oben aufgeführten Weiterverwendungen des Materials die Einwilligung des jeweiligen Rechteinhabers einzuholen.

Weitere Details zur Lizenz entnehmen Sie bitte der Lizenzinformation auf http://creativecommons.org/ licenses/by/4.0/deed.de.

Dr. Alexis von Mirbach ist wissenschaftlicher Mitarbeiter an der Ludwig-Maximilians-Universität in München. 